\title{
Bundeswehr-Reform: kleine Schritte und ein großer Sprung
}

von Wilfried von Bredow

\begin{abstract}
Anders als in der Außenpolitik nahmen die deutsche Öffentlichkeit und die politische Führung des Landes die neuen sicherheitspolitischen Herausforderungen über einen längeren Zeitraum hinweg als fremd oder gar unheimlich wahr. Die Auslandseinsätze der Bundeswehr wurden meist als kampflose Friedens- und Stabilisierungsmissionen dargestellt, die notwendige Reform der Streitkräfte wurde entsprechend nur als eine Art „Marathon der kleinen Schritte“ in Angriff genommen - mit bislang mageren Ergebnissen. Der nachfolgende Artikel beschreibt die konzeptionelle Entwicklung im verteidigungspolitischen Bereich und analysiert die derzeit vorliegenden Vorschläge zur Wehrreform. Dabei ist festzustellen, dass die angeregten Veränderungen weder zu den ersehnten Einspareffekten führen dürften noch die Frage nach der Rekrutierung des Personals für eine zukünftige Freiwilligenarmee zufriedenstellend beantworten.
\end{abstract}

In contrast to their acceptance of new foreign policy challenges, the German public and its political representatives have never felt at ease with the parallel developments in security policy. The overseas missions of the German military were mostly framed as combat-free endeavours of peacekeeping and stabilisation, necessary reforms of the Armed Forces have accordingly only been addressed as a "marathon of small steps" with sobering results. The following article describes the conceptual development in the area of defence policy and seeks to analyse the current proposals for military reform, leading to the conclusion that these reforms will hardly lead to the desired fiscal savings while the question of recruitment for any future personnel structure based on professional volunteers (in place of a conscription-based force) remains largely unaddressed.

Zwanzig Jahre nach dem Ende des Ost-West-Konflikts und den dadurch in Gang gesetzten dramatischen Veränderungen der sicherheitspolitischen Landschaft in Europa und auf anderen Kontinenten hat in Deutschland gegenwärtig erstmalig eine grundlegende und umfassende Debatte über die Prioritäten der eigenen Sicherheitspolitik und über die entsprechend diesen Prioritäten umzugestaltende Bundeswehr begonnen. Grundlegend und umfassend - damit ist gemeint, dass außen-, sicherheits- und gesellschaftspolitische Aspekte mit militärstrategischen und organisations-administrativen Perspektiven der Bundeswehr-Entwicklung zusammengeführt und nicht länger isoliert voneinander, vielmehr in ihren wechselseitigen Bezügen debattiert werden. 
Von manchen auswärtigen Beobachtern wird den Deutschen in Politik und Wirtschaft häufig unterstellt, sie würden vor wichtigen Entscheidungen erst einmal ein längerfristig orientierendes Gesamtkonzept anstreben. ${ }^{1}$ Wenn es nur immer so wäre! Die Auseinandersetzungen um die Anpassung der Bundeswehr an die veränderten sicherheitspolitischen Herausforderungen und die im Laufe der letzten Jahre dazu getroffenen Entscheidungen haben eher StückwerksCharakter. Dabei muss allerdings berücksichtigt werden, dass auf diesem Politikfeld ein ausgeprägtes Beharrungsvermögen in Politik, Militärführung und ziviler Gesellschaft existiert.

Das hat seine besonderen und auch nicht einfach vom Tisch $\mathrm{zu}$ wischenden Gründe. Um die gegenwärtige, weit ausgreifende und große Reformschritte annoncierende Politik der Bundesregierung und das öffentliche Echo darauf klarer beurteilen zu können, bietet sich ein scheinbar etwas umständliches Verfahren an. Zunächst sollen in diesem Beitrag die wichtigsten, seit dem Ende des OstWest-Konflikts sich vollziehenden Veränderungen in der internationalen Sicherheitslandschaft ${ }^{2}$ skizziert werden. Aus diesen Veränderungen, die oftmals erst mit Verzögerungen wahrgenommen wurden, ergaben und ergeben sich neue Anforderungs-Profile für die Bundeswehr und für die Soldaten, sozusagen das Ziel und der Sinn aller organisatorischen und professionellen Anpassungsmaßnahmen. Sie werden im zweiten Kapitel in gedrängter Form angesprochen. Danach werden die einzelnen Stufen oder Schritte bei der Reform der Bundeswehr im Überblick vorgestellt. Bei der Bewertung dieses Prozesses darf nicht übersehen werden, dass er für die Angehörigen der Streitkräfte große Umstellungen mit sich brachte, die gewiss nicht immer einfach zu bewältigen waren. Das Tempo der Reformen erscheint denen, die sie durchführen müssen, gewiss rascher als dem Beobachter von außen.

Der Horizont und die Dynamik der jüngsten Reform-Debatte um die Zukunft der Bundeswehr werden vor allem von zwei Entwicklungen bestimmt, der Finanzkrise und dem, was man den Afghanistan-Schock nennen könnte. Während die Finanzkrise und die auf sie folgenden Sparbeschlüsse der Bundesregierung einen generellen Einsparungs-Druck auf den Bundeshaushalt erzeugt haben, ist durch

1 Vgl. etwa Smyser, W. R.: How Germans Negotiate. Logical Goals, Practical Solutions, Washington D.C., 2003.

2 Die Metapher von der Sicherheitslandschaft meint das Insgesamt von Risiken, Gefahren und Bedrohungen der Sicherheit aus der Perspektive der staatlichen Akteure und internationaler Organisationen wie den Vereinten Nationen plus das Insgesamt von sicherheitspolitischen Bündnissen (wie der NATO), internationalen Regimen (wie der Internationalen Atomenergie-Behörde) und Abkommen. 
die kriegerischen Verwicklungen der Bundeswehr in Afghanistan einer Reihe von euphemistischen Vorstellungen über die programmatische Kriegs-Ferne der Bundeswehr bei internationalen Einsätzen der Boden entzogen worden. So wird das Jahr 2009 in die Geschichte der Bundeswehr als tiefer Einschnitt und als Beginn folgenreicher Richtungsänderungen in ihren Reform-Planungen eingehen. Nur vor diesem Hintergrund lassen sich die jüngsten Reform-Vorschläge von Verteidigungsminister $z u$ Guttenberg (CSU) angemessen verstehen. Über diese Vorschläge ist bei Abfassung dieses Beitrags das letzte Wort noch nicht gesprochen. Mit Änderungen in Einzelfragen ist zu rechnen, kaum hingegen mit dem Abblasen der Reformen. Diese haben im Übrigen nicht nur Auswirkungen auf die Organisation der Streitkräfte, sondern für die Gesellschaf insgesamt.

Der Tenor meiner Ausführungen ist überwiegend reform-freundlich, manchmal indes auch kritisch-ungeduldig. Denn der Lern- und Anpassungsprozess, welche der Bundeswehr verordnet wurde und wird, hätte mutiger kalkuliert werden können.

\section{Die Veränderung der internationalen Sicherheitslandschaft nach 1990}

Im Ost-West-Konflikt nach 1945 beherrschte die (asymmetrische) nukleare Konfrontation der beiden Führungsmächte der feindlichen Lager das militärstrategische Denken und Handeln. Allerdings nicht ausschließlich, denn in den Entkolonialisierungs-Kriegen dieser Ära kamen sehr erfolgreich auch Methoden der Partisanen- und Guerilla-Kriegsführung zur Anwendung. Diese Art der Kriegsführung hat vieles mit den heutigen Konstellationen sogenannter neuer Kriege gemeinsam. ${ }^{3}$ Die Sicherheitspolitik der Bundesrepublik Deutschland war in dieser Zeit fest in das westliche Konzept der nuklearen Abschreckung eingebunden. Die Bundeswehr bereitete sich auf Kriegsszenarien vor, in denen es um die Verteidigung des eigenen Territoriums gegen einen Angriff aus dem Osten ging. Glaubwürdig demonstrierte Verteidigungsbereitschaft galt als notwendiges Element der Abschreckung. Die Soldaten übten das Kämpfen, um das Geübte nicht anwenden zu müssen. Zum Glück kam es wegen oder unabhängig von der Abschreckung nicht zu einem Angriff auf das Territorium der Bundesrepublik.

3 Vgl. Münkler, H.: Die neuen Kriege, Reinbek, 2002; ders.: Der Wandel des Krieges. Von der Symmetrie zur Asymmetrie, Weilerswist, 2006. 
An die bipolare Grundstruktur der internationalen Sicherheitslandschaft mit ihren eindeutigen Grenzverläufen (Eiserner Vorhang, Bambusvorhang) waren Unsicherheitszonen angedockt. In diesen Zonen fanden Kriege aus lokalen und regionalen Ursachen statt, aber nicht selten wurden sie von den Weltmächten kontrolliert und instrumentalisiert.

Als solche Eindämmungs- und Kontrollmechanismen wegfielen, weiteten sich diese Unsicherheitszonen aus. Zugleich verloren lokale und regionale Kriegsursachen und -anlässe mehr und mehr ihre geographische Begrenztheit. Handlungs- und Kommunikationsakteure können praktisch jederzeit potentiell globale spill over-Effekte bewirken. Militärische Konfrontationen entwickeln sich aus inner-ethnischen und religiös aufgeheizten Konflikten. Hunger und Elend ganzer Gesellschaften als Folge von Naturkatastrophen, beschleunigtem Bevölkerungswachstum oder (besonders häufig) der verantwortungslosen Politik von schwachen oder korrupten Staatsführungen können zu regionalen Unruhen führen, die sich rasch ausbreiten. Die dadurch ausgelösten Migrationsströme bringen auch die Nachbarstaaten in wirtschaftliche Schwierigkeiten. Es findet gleichsam ein Export von Instabilität statt. Für nicht-staatliche Akteure wie Terrornetzwerke und kriminelle Organisationen (Drogenschmuggler, Menschenhändler, Piraten) eröffnen sich so zahlreiche Handlungsmöglichkeiten. Bei konventionellen Waffen gibt es einen regen, teils legalen, teils illegalen internationalen Handel. Er trägt dazu bei, dass überall dort, wo Konflikte gewaltsam ausgetragen zu werden drohen, die Mittel zur Eskalation rasch und relativ problemlos in die Hände der Konfliktparteien gelangen. Wer bezahlen kann, bekommt Waffen geliefert. Wer nicht bezahlen kann, bekommt sie oft genug von Sympathisanten gespendet.

Internationale Bandenkriminalität und politisch oder anderweitig motivierter internationaler Terrorismus können die innere Sicherheit von Staaten erheblich gefährden. Da die Drahtzieher außer Landes residieren und Staatsgrenzen ihre eigenen Aktivitäten kaum, wohl aber die der Regierungen beeinträchtigen, ist hier die Unterscheidung zwischen innerer und äußerer Sicherheit hinfällig geworden. Das technische Wissen und die Bestandteile, die benötigt werden, um Massenvernichtungswaffen zu bauen, sind auf einem globalen schwarzen Markt ohne große Schwierigkeiten zu erwerben. Solche Waffen in der Verfügungsgewalt von verantwortungslosen Staatsführern oder im Besitz von Terroristen oder Kriminellen stellen ein großes Bedrohungspotenzial dar. Denn sie sind schwer zu entdecken und einfach an beliebige Orte zu transportieren. Fortschreitende Verknappung lebenswichtiger Ressourcen wie Rohöl oder Wasser belebt demgegenüber das nur scheinbar obsolet gewordene Muster zwischenstaatlicher Kriege. 
Sicherheitspolitik muss künftig nicht nur auf diese Bedrohungen reagieren, sondern außerdem berücksichtigen, dass sich auch die Art und Weise der Kriegsführung verändert hat. Die Rüstungstechnologie entwickelt sich auf allen Ebenen weiter, bei den hochtechnischen Großsystemen ebenso wie bei den Kleinwaffen.

Viele kriegerische Konflikte werden nicht nur durch staatliche Streitkräfte mit völkerrechtlich eindeutig als Kombattanten ausgewiesenen Soldaten, sondern auch durch Banden, Söldner und Techno-Partisanen, kleinen Gruppen mit verdeckten politischen Zielen und dem Zugang zu modernsten Waffensystemen, ausgetragen werden. Es findet eine Verschmelzung von konventioneller und Guerilla-Kriegsführung statt.

Die von manchen Experten als revolutionär bezeichnete Entwicklung der elektronischen Informations- und Führungssysteme und die damit verbundene Technisierung des Krieges (revolution in military affairs) hat keineswegs einen „klinisch sauberen" Krieg zur Folge. Information warfare ist in den Kriegen der letzten Jahre zu einem wichtigen Bestandteil der Kriegsführung geworden, wobei die Medien der eigenen Seite und die des Gegners jeweils als Instrumente zur Unterstützung der eigenen Ziele eingesetzt werden. Seine Bedeutung wird weiter zunehmen. Mit dem Begriff cyber war werden außerdem neue Gefahren für postmoderne Gesellschaften bezeichnet, deren Infrastruktur in wachsendem Maße von der Informations- und Kommunikationstechnologie abhängig ist. Kann man sie lahm legen, werden wichtige Funktionszusammenhänge in diesen Gesellschaften unterbrochen - mit unabsehbaren Folgen.

Die aufgeführten Bedrohungen machen vor allem eines deutlich: Die Verletzlichkeit ziviler Gesellschaften einschließlich und trotz ihrer Streitkräfte hat sich drastisch erhöht. Herkömmliche Konzepte und Instrumente der Sicherheitspolitik allein bieten keinen wirksamen Schutz.

\section{Ein Marathon der kleinen Schritte}

Im Fall des vereinten Deutschlands kommt hinzu, dass viele Regierungen und nicht zuletzt die Generalsekretäre der Vereinten Nationen von ihm vermehrte Aktivitäten zur Aufrechterhaltung und Gestaltung der Weltordnung erwarteten nicht nur einen zivilen Beitrag in Form von Entwicklungshilfe und Beiträgen für das Funktionieren internationaler Organisationen, sondern auch einen militärischen Beitrag in Form von Bundeswehr-Kontingenten für Friedens- und Stabilisierungsmissionen, humanitäre Interventionen, die Überwachung von Demokratisierungsprozessen und die Schulung einheimischen Militärpersonals in der 
Wiederaufbauphase nach einem gewaltsamen Konflikt. Auf solche Aufgaben waren weder die deutschen Sicherheitspolitiker noch die Bundeswehr vorbereitet. Wie hätte das auch sein können? Vor 1990 hat sich die Bundeswehr an derlei niemals beteiligt (mit Ausnahme reiner Katastrophenhilfe). Sie durfte das nicht, und die Bundesregierungen wollten auch, dass sie das nicht durfte. Das gängige und ein großes Stück weit internalisierte Argument lautete: Das Grundgesetz verbietet die Teilnahme an solchen Einsätzen. ${ }^{4}$ Die politische Absicht zeugte durchaus von Urteilskraft; die verfassungsrechtliche Begründung hingegen stand auf schwachen Füßen. Nach dem dramatischen Wandel des internationalen Systems zu Beginn der 1990er Jahre stand aber auch eine Korrektur des sicherheitspolitischen Horizonts der Bundesrepublik Deutschland an - und damit eine Neufassung der Aufgaben ihres militärischen Instruments, der Bundeswehr. ${ }^{5}$

\section{Das Weißbuch 1994}

Die im „Weißbuch zur Sicherheit der Bundesrepublik Deutschland und zur Lage und Zukunft der Bundeswehr" vom April 1994 vorgenommene Lageanalyse steht noch weitgehend unter dem Eindruck jener Veränderungen von Sicherheitspolitik und Bundeswehr, die durch den Vereinigungsprozess angestoßen wurden. Aber zugleich werden auch einige Zielvorstellungen für die nächsten Jahre angesprochen. Verteidigungsminister Volker Rühe (CDU) unterstreicht in seinem Vorwort die Absicht, gemeinsam mit Frankreich die europäische Sicherheits- und Verteidigungspolitik voranzubringen.

Die Gefahr eines großangelegten und existenzbedrohenden Angriffs auf das Territorium Deutschlands wird in der Lagebeurteilung des Weißbuchs als überwunden bezeichnet. Dieser Topos taucht seither in allen grundlegenden sicherheitspolitischen Dokumenten der Bundesregierungen auf, allerdings ohne dass lange Zeit ernsthaft Konsequenzen daraus gezogen werden. Jedoch, heißt es weiter, seien andere Regionen in Europa und in anderen Teilen der Welt durch Krieg, Unmenschlichkeit und Unterdrückung bedroht. Solche Entwicklungen würden nicht nur den Frieden in der Völkergemeinschaft, sondern auch die Lebensgrundlagen der gesamten Menschheit gefährden. Deshalb müssten Konflikt-

4 Außerhalb ihres Abschreckungs- und territorialen Verteidigungsauftrags gab es für die Bundeswehr allerdings zahlreiche (nichtmilitärische) humanitäre Hilfseinsätze. Eine Aufstellung findet sich bei Chiari, B./Pahl, M. (Hg.): Auslandseinsätze der Bundeswehr, Paderborn, 2010, 297-301.

5 Das war den sicherheitspolitischen Experten und der Bundeswehr-Führung selbstverständlich klar. Vgl. etwa das Buch des damaligen Generalinspekteurs der Bundeswehr: Naumann, K.: Die Bundeswehr in einer Welt im Umbruch, Berlin, 1994, 123-169. 
verhütung und Krisenbewältigung im erweiterten geographischen Umfeld Deutschlands in Zukunft im Vordergrund der Sicherheitsvorsorge stehen.

Aus dieser Lageanalyse ergibt sich für das Weißbuch 1994, dass die angemessene und wirkungsvolle Teilnahme an internationalen KrisenbewältigungsMissionen zur zweiten Hauptfunktion der Bundeswehr werden muss. Die erste Hauptaufgabe bleibt Landes- und Bündnisverteidigung. Aber wegen der rund um das eigene Territorium entscheidend verbesserten Sicherheitslage erscheint dies schon hier und mehr noch in den Reform-Dokumenten der folgenden Jahre als eine rituelle Beschwörung der sicherheitspolitischen Vergangenheit, allerdings eine mit hohem Beliebtheitsgrad.

\section{Die Entscheidung des Bundesverfassungsgerichts vom 12. Juli 1994}

Die Frage war zu diesem Zeitpunkt noch, wie eine Umstellung der Bundeswehr, um sie für neue Aufgaben funktionsfähig zu machen, einzuleiten und verfassungsrechtlich abzustützen sei. Denn von verschiedener Seite lagen beim Bundesverfassungsgericht in Karlsruhe Klagen gegen Einsätze von BundeswehrKontingenten auf dem Balkan (ab Juni 1992) und in Somalia (ab August 1993) vor.

Mitte Januar 1993 konstituierte sich die Gemeinsame Verfassungskommission des Deutschen Bundestages und des Bundesrates (GKV). Sie sollte herausfinden, wo und wie das Grundgesetz als Folge der Vereinigung Deutschlands zu überarbeiten sei. In den Parteien gab es auch eine Reihe von Überlegungen zur Neufassung oder Präzisierung derjenigen Artikel, die den Einsatz der Bundeswehr außerhalb des NATO-Territoriums betrafen. Ende Oktober 1993 legte die Kommission ihren insgesamt ziemlich mageren Abschlussbericht vor. Eine Empfehlung der Kommission an die Parlamente zu diesem Beratungsgegenstand sucht man darin vergeblich. Stattdessen wurde die Öffentlichkeit darauf verwiesen, die Entscheidung des Bundesverfassungsgerichts $\mathrm{zu}$ den insgesamt vier Klagen abzuwarten. ${ }^{6}$ Das Bundesverfassungsgericht stützte sich in seiner Entscheidung vom 12. Juli 1994 vor allem auf den Art. 24, Abs. 2 GG: „Der Bund kann sich zur Wahrung des Friedens einem System gegenseitiger kollektiver Sicherheit einordnen; er wird hierbei in die Beschränkungen seiner Hoheitsrechte einwilligen, die eine friedliche und dauerhafte Ordnung in Europa und zwischen den Völkern der Welt herbeiführen und sichern." Damit seien, so das Gericht, 
auch Einsätze der Bundeswehr erlaubt, die im Rahmen und nach den Regeln eines solchen Systems gegenseitiger kollektiver Sicherheit stattfinden. Das Gericht verneinte ausdrücklich einen Widerspruch zwischen dieser Auslegung des Art. 24 (2) und dem Artikel 87 a GG, dessen erster Satz lapidar lautet: „Der Bund stellt Streitkräfte zur Verteidigung auf.“ Außerdem legte das Gericht der Bundesregierung die Pflicht auf, vor jedem Einsatz der Bundeswehr die konstitutive Zustimmung des Deutschen Bundestages einzuholen.

Diese Karlsruher Entscheidung wurde in der Öffentlich sogleich als eine weitreichende Strukturentscheidung wahrgenommen. Sie beendete mit einem Schlag die in den frühen 1990er Jahren immer einmal wieder aufflackernde Debatte über Legalität und Legitimität von Auslandseinsätzen der Bundeswehr im Kontext von multinationalen Friedens- und Stabilisierungsmissionen. Sie öffnete das Tor für grundlegende Reformen des Auftragsprofils der Bundeswehr, ihrer Umgestaltung, Ausstattung und der Ausbildung ihrer Soldaten.

\section{Der Bericht der Weizsäcker-Kommission und das „Eckpfeiler"-Papier 2000}

Weißbuch 1994 und BVerfG-Entscheidung 1994 illustrieren das eigentümliche Reform-Dilemma der deutschen Sicherheitspolitik und die sich daraus ergebenden Schwierigkeiten für die Organisation der Streitkräfte. Denn weil aus der Formulierung des Art. 87 a GG mit schwer zu verschleiernder Klarheit hervorgeht, dass der Zweck der Bundeswehr in der Verteidigung besteht, müssen alle Missionen und Einsätze von deutschen Soldaten irgendwie als Verteidigungsmaßnahmen wahrgenommen werden können. Nur ganz allgemein und abstrakt lässt sich das auch für Einsätze behaupten, die zur Wahrung des Friedens und zur Herstellung einer friedlichen und dauerhaften Ordnung in Europa und zwischen den Völkern der Welt beitragen. Im konkreten Einzelfall kann das jedoch sehr schwierig werden, wie sich bald erwies.

In der Endphase des NATO-Einsatzes zur Verhinderung ethnischer Säuberungen durch serbische Truppen an den Kosovo-Albanern legte der Amtsnachfolger Volker Rühes, Rudolf Scharping (SPD), eine „Bestandsaufnahme“ der Sicherheitspolitik und Bundeswehr vor. Darin findet sich der Satz „Landesverteidigung ist zum unwahrscheinlichsten Einsatzfall geworden. “ Wenig später heißt es dann: „Landes- und Bündnisverteidigung bleibt gleichwohl wesentliche Aufgabe der 
Streitkräfte.“ ${ }^{7}$ Diesem eklatanten Widerspruch sollte mit Vorschlägen einer von Minister Scharping eingesetzten unabhängigen Kommission „Gemeinsame Sicherheit und Zukunft der Bundeswehr" abgeholfen werden. Nach ihrem Vorsitzenden wurde sie auch „Weizsäcker-Kommission“ genannt. Im Frühjahr 1999 trat sie zusammen, Ende Mai 2000 präsentierte sie ihren Abschlussbericht.

Die Kommission sollte Reform-Vorschläge für den Umfang, die Struktur, die Bewaffnung und Ausrüstung der Bundeswehr machen, damit sie erstens auf ihre „wahrscheinlichste Aufgabe“ vorbereitet sein würde, nämlich die Teilnahme an Einsätzen der Krisenvorsorge ${ }^{8}$ und Krisenbewältigung, damit sie zweitens besser zur wirksamen Zusammenarbeit mit Partnern in der NATO, der Europäischen Union, den Vereinten Nationen und der OSZE befähigt wird und drittens sich soweit wie möglich europäisieren kann. ${ }^{9}$

Dieser sehr weitgehende Reformauftrag speiste sich aus der anfänglichen politischen Dynamik der neuen rot-grünen Bundesregierung unter Kanzler Gerhard Schröder. Auch gab es zu diesem Zeitpunkt bereits eine Reihe von internationalen sicherheitspolitischen Dokumenten, die gewissermaßen als Richtschnüre für die deutschen Reformbemühungen gelten konnten: das weiterentwickelte Strategie-Konzept der NATO vom April 1999, die WEU-Erklärung von Bremen im Mai 1999, die Kölner Erklärung des Europäischen Rates über die Verstärkung der Gemeinsamen Europäischen Sicherheits- und Verteidigungspolitik (ESVP) vom Juni 1999 und schließlich die Beschlüsse desselben Gremiums vom Dezember 1999 in Helsinki über die bis spätestens 2003 zu erfolgende Aufstellung einer Schnellen Eingreiftruppe der Europäischen Union. ${ }^{10}$

Die Weizsäcker-Kommission empfahl der Bundesregierung einen Katalog von Maßnahmen, darunter unter anderem die Umgliederung der Streitkräfte, um sie einsatzfähiger zu machen, die Reduzierung der Friedensstärke auf 240.000 Soldaten, die Förderung der Multinationalisierung der Einsatzkräfte, die Moderni-

7 Bundesministerium der Verteidigung: Bestandsaufnahme. Die Bundeswehr an der Schwelle zum 21. Jahrhundert, Bonn, 1999, 25.

8 Krisenvorsorge mit militärischen Mitteln, das ist auch so ein abstrakter und auf solcher Ebene nur nützlich erscheinender Begriff mit einer common sense-Aura (,,Vorsorgen ist besser als Heilen“). Fast alles, was in der Politik mit Prävention zusammenhängt (im letzten Jahrzehnt ein recht populäres Konzept), überfordert sie aber.

9 Gemeinsame Sicherheit und Zukunft der Bundeswehr: Bericht der Kommission an die Bundesregierung, Bonn, 2000, 14.

10 Dass nicht alles, was in diesen Dokumenten aufgeführt worden ist, längeren Bestand hatte oder umgesetzt werden konnte, steht auf einem anderen Blatt. Wie viele andere sicherheitspolitische Entwicklungen wurden auch diese durch die Ereignisse vom 11. September 2001 aus ihren Bahnen gelenkt. 
sierung der Ausrüstung, die Privatisierung von Dienstleistungen und die gleichberechtigte Zulassung von Frauen in den Streitkräften. Alles zusammen ergab ein behutsames Konzept für die „Erneuerung der Bundeswehr von Grund auf“. Das hätte so kommen können, hätte nicht der Minister kurz nach Kenntnisnahme der Kommissions-Empfehlungen ein sogenanntes „Eckpfeiler“-Papier vorgelegt, das in seinem Ministerium erarbeitet worden war und in dem diese Empfehlungen an wichtigen Stellen abgeschwächt wurden.

\section{Die Verteidigungspolitischen Richtlinien 2003 und die Neu-Konzeption der Bundeswehr 2004}

Als einer der sperrigsten Stolpersteine für eine zügige Reform der Bundeswehr lässt sich ein unrealistisches Optimierungskonzept ausmachen. Ihm zufolge sollte der neue Interventionsauftrag der Bundeswehr zum Zwecke der Deeskalierung lokaler Konflikte im Rahmen multinationaler Friedens- und Stabilisierungsmissionen bruchlos in den alten territorialen Verteidigungs-Auftrag der Bundeswehr integriert werden. Gewiss ist es sinnvoll, wegen der veränderten Weltlage mit ihrer wenig übersichtlichen Sicherheits-Landschaft nicht sogleich und ohne eine Art Rückversicherung alle „klassischen“ militärischen Fähigkeiten zur Verteidigung des eigenen und des Territoriums der Verbündeten zur Disposition zu stellen. Jedoch geht es um mehr als nur rhetorische Anpassung und setzt mehr als nur postulierte Reformen voraus, wenn man sich verpflichtet, eine Reihe von (jedenfalls für die Bundeswehr) neuen militärischen Fähigkeiten aufzubauen. Anders gesagt: es geht um Prioritätensetzung, und das ist das Gegenteil jener Sowohl-als auch-Haltung, von der sich die politische und militärische Führung der Streitkräfte über fast zwei Jahrzehnte hinweg nicht verabschieden wollte.

Nirgends kommt diese Haltung unverblümter zum Ausdruck als in dem Satz von Scharpings Amtsnachfolger Peter Struck (SPD) auf einer Pressekonferenz Ende 2002, wonach Deutschlands Sicherheit auch am Hindukusch verteidigt würde. Dieser Satz, von Journalisten schon einmal als „legendär“ oder gar als „StruckDoktrin“ bezeichnet, hätte eigentlich als Ausgangspunkt einer umfassenden Bundeswehrreform-Debatte dienen können, gerade weil er so eigentümlich verschwurbelt ist. Aber damals, in der Frühphase des Afghanistan-Einsatzes der Bundeswehr, kam es dazu nicht.

Minister Struck unternahm im Mai 2003 einen erneuten Anlauf, um die Bundeswehr-Reform voranzubringen. Die „Verteidigungspolitischen Richtlinien“ hätten eigentlich sicherheitspolitische Richtlinien heißen müssen. Aber dann hätte das 
Auswärtige Amt sie mitzeichnen müssen, wogegen es sich sträubte. In den neuen Richtlinien wird festgestellt: „Eine Gefährdung deutschen Territoriums durch konventionelle Streitkräfte gibt es derzeit und auf absehbare Zeit nicht. Das Einsatzspektrum der Bundeswehr hat sich grundlegend gewandelt. " ${ }^{11}$ Diese Einsicht hatte sich ja nun weitgehend durchgesetzt. Folgerungen daraus kündigten die „Grundzüge der Konzeption der Bundeswehr“ vom August 2004 an: „Die Bundeswehr benötigt nach Einsatzbereitschaft und Fähigkeiten konsequent differenzierte Streitkräfte. Sie müssen schnell, wirksam, durchsetzungsfähig und durchhaltefähig gemeinsam mit den Streitkräften anderer Nationen eingesetzt werden können. Dazu ist ein Fähigkeitsprofil erforderlich, das sechs miteinander verzahnte Fähigkeitskategorien umfasst: Führungsfähigkeit, Nachrichtengewinnung und Aufklärung, Mobilität, Wirksamkeit im Einsatz, Unterstützung und Durchhaltefähigkeit, Überlebensfähigkeit und Schutz. “12 Diese Formulierungen gehen großenteils auf den damaligen Generalinspekteur der Bundeswehr Wolfgang Schneiderhan zurück. Sie sind zwar immer noch relativ allgemein gehalten, werden aber in weiteren Teilen des Dokuments operationalisiert. Schneiderhan war es auch, der für den Reformprozess der Bundeswehr einen griffigen Oberbegriff einführte, der in angelsächsischen Staaten seit längerem zur Kennzeichnung von deren Streitkräfte-Reformen benutzt wurde: Transformation. Mit diesem Begriff sowie dem der ,vernetzten Sicherheit“ ließ sich zumindest das Skelett eines Gesamtkonzeptes für die Bundeswehr-Reform andeuten.

\section{Das Weißbuch 2006}

Transformation ist im Weißbuch 2006 bereits zu einem geläufigen Begriff geworden. Ihr ist ein besonders Kapitel gewidmet. „Transformation erfordert die Bereitschaft und den Willen zur Umgestaltung. Ohne die nachhaltige Bereitschaft zur Veränderung kann die Transformation der Bundeswehr nicht gelingen. “13 Obwohl in diesem Jahr nicht mehr Peter Struck (SPD), sondern Franz Josef Jung (CDU) Verteidigungsminister ist, gibt es gegenüber der NeuKonzeption von 2004 kaum Veränderungen in den Inhalten und dem Tempo des Reformprozesses der Bundeswehr. Allerdings hat sich der ErfahrungsHintergrund der Bundeswehr im Stabilisierungs-Einsatz in Afghanistan seit 2005

11 Bundesministerium der Verteidigung: Verteidigungspolitische Richtlinien, Berlin, 2003, 4.

12 Bundesministerium der Verteidigung: Grundzüge der Konzeption der Bundeswehr, Berlin, 2004, 17.

13 Bundesministerium der Verteidigung: Weißbuch 2006 zur Sicherheitspolitik Deutschlands und zur Zukunft der Bundeswehr, Berlin, 2006, 102. 
schon ein Stück weit verdüstert. Damit wird die Notwendigkeit zur Prioritätensetzung bei Ausbildung und Ausrüstung der Truppe wird immer unabweisbarer. Im Weißbuch 2006 wird deshalb betont: „Der Aufbau neuer, zukunftsträchtiger Elemente und der Abbau nicht mehr benötigter Strukturen dienen der konsequenten Einsatzorientierung der Bundeswehr ... Die große Anzahl der noch bestehenden nichtaktiven Truppenteile wird bis 2010 deutlich verringert. “14

Seit 1990 befindet sich, kann man als Zwischenbilanz resümieren, die Bundeswehr in einem quasi-permanenten Umwandlungsprozess, was ihren Umfang betrifft, ihre Aufgaben und die Ausbildung ihrer Soldaten. Dennoch aber, so erkennt man im Rückblick ziemlich deutlich, ist bei diesem Vorgang weniger erreicht worden, als es aus heutiger Sicht wünschbar gewesen ist. Weil man sich nur ganz kleine Schritte zutraute, ist man auf der Marathonstrecke trotz heftiger Anstrengungen nicht sehr weit gekommen.

Das liegt vor allem an drei Gründen. Erstens hat sich die Sicherheitspolitik Deutschlands zu langsam und gewissermaßen über-vorsichtig auf die Erfordernisse der neuen Sicherheits-Landschaft in und jenseits von Europa eingelassen. Zweitens hat die militärische Führung der Bundeswehr kein erfolgreiches Rezept entwickelt, um inner-organisatorisches Beharrungsvermögen und Schwierigkeiten mit der eigenen Bürokratie zu überwinden. Der dritte Grund wiegt möglicherweise am schwersten: Es ist nicht gelungen, eine aufgeklärte und informierte öffentliche Debatte über die Zukunft der Bundeswehr sowie über ihre neuen militärischen Aufgaben, aber auch über die damit verbundenen Erschwernisse des Soldatenberufs in Gesellschaft und Öffentlichkeit in Gang zu bekommen. Dazu brauchte es erst eines Anstoßes von außen.

\section{Der Afghanistan-Schock}

Ohne die Erfahrungen, welche der Bundeswehr seit 2005/06 bei ihrem ISAFEinsatz $^{15}$ in Afghanistan aufgenötigt wurden, würde sich die BundeswehrReform vermutlich nach wie vor mit relativ kleinen Schritten vortasten. Zur

14 Ebd., 93.

15 ISAF ist das Akronym für International Security Assistance Force. Diese Hilfsmission zum Schutz des Wiederaufbaus staatlicher und gesellschaftlicher Strukturen Afghanistans wurde Ende 2001 beschlossen und ist durch eine Resolution der Vereinten Nationen legitimiert. Organisatorisch steht sie unter der Führung der NATO. Bei einer zweiten Mission, der Operation Enduring Freedom (OEF), geht es um die direkte Verfolgung und Ausschaltung von Terroristen. An dieser weiträumigen Mission waren in Afghanistan zwischen 2001 und 2008 auch Bundeswehr-Soldaten vom Kommando Spezialkräfte (KSK) in geringer Zahl beteiligt. 
Erinnerung: Das deutsche Engagement in Afghanistan war anfangs von mehreren Motiven geleitet. Der Einsatz sollte die Solidarität Deutschlands mit den Vereinigten Staaten nach dem Terrorangriff vom 9. September 2001 dokumentieren; später dann fungierte er als eine Art Kompensation für die Nichtbeteiligung an der amerikanischen Intervention im Irak. Außerdem sollte demonstriert werden, dass man den Kampf gegen den Terrorismus erfolgreicher anlegen kann, als es die Vereinigten Staaten im Irak taten, nämlich nicht durch einen „brachialen Militäreinsatz, sondern durch ein kluges Zusammenspiel von zivilen und militärischen Elementen““16. Das war recht hochmütig und ein Ausdruck einer Art von politischem Narzismus.

Aus deutscher Perspektive, aber auch aus der anderer europäischer Länder (und selbstverständlich ebenso des Pentagon) war klar, dass die entscheidende Aufgabe in Afghanistan die Stabilisierung von Staat und Gesellschaft war: Aufbau funktionierender Verwaltungsstrukturen, Sicherung von Demokratie und Menschenrechten, aber ohne die unvermittelte Übertragung westlicher Vorstellungen dazu auf das Land, Abbau der Korruption, Stärkung der Wirtschaft. Aus einem failed state sollte mittels state building wieder ein verlässlicher Akteur der internationalen Politik werden. ${ }^{17}$ Für all das brauchte es in erster Linie zivile Mittel. Streitkräfte erschienen nach dem Fall der Taliban nur nötig, um diese eigentlichen Aufgaben quasi polizeilich abzusichern. Viele Vertreter der zahllosen Nichtregierungs-Organisationen, die nun im Lande mit Hilfs- und Wiederaufbauprogrammen agierten, lehnten sogar solchen Schutz grundsätzlich ab.

Der Aufbau des Landes kam nur schleppend voran, und vor allem in den südlichen und südwestlichen Provinzen verstärkte sich ab 2005 der Widerstand zurückkehrender Taliban-Kämpfer, welche die kaum kontrollierbare Grenze zwischen Pakistan und Afghanistan geschickt für ihre ,asymmetrische Kriegsführung" nutzbar machten. Dass zu dieser Zeit die Bundesregierung auf den Sonderkonditionen für ihren Einsatz im Norden Afghanistans beharrte und annahm, hier käme es wegen der eigenen Popularität unter den Afghanen nicht zu bewaffneten Konflikten, beruht auf Wahrnehmungsfehlern in den zuständigen Ministerien und im Bundeskanzleramt.

16 Neue Zürcher Zeitung, 5./6. September 2009.

17 Dass diese Aufgabe erheblich schwieriger zu bewältigen ist, als damals angenommen wurde, ist im Rückblick ganz deutlich zu erkennen. Trotzdem ist es vielleicht nicht ganz fair gegenüber den damaligen Protagonisten solcher Pläne, sie als gänzlich unrealistische und verblendete Polit-Träumer abzutun. 


\section{Mangelhafte Vorbereitung}

Die Vertreibung der Taliban und damit ein „Regimewechsel“ gingen vergleichsweise rasch über die Bühne, und den ISAF-Truppen wurde zumeist sehr freundlich und in der Erwartung begegnet, dass der Bürgerkrieg nun zu Ende und die Zeit für einen Neuanfang gekommen seien. Diese positive Grundstimmung gegenüber den Fremden und der von ihnen, aber auch über einheimische Legitimations-Mechanismen gestützten Regierung in Kabul unter Präsident Karzai verflachte allerdings in den folgenden Jahren. Der Neuanfang kam über vielfache Versprechungen kaum hinaus. ${ }^{18}$

Warum das so kam, ist eine komplizierte und langwierige Geschichte, die an anderer Stelle zu erzählen ist. Hier mag der Hinweis genügen, dass sich trotz vielfältiger Bemühungen von Staaten und nichtstaatlichen Organisationen die Lebensbedingungen für einen sehr großen Teil der afghanischen Bevölkerung nicht verbesserten. Das ist zwar nicht der einzige, aber der Hauptgrund dafür, dass sich seit 2005 der Widerstand gegen die ausländischen Truppen und die Regierung in Kabul allenthalben verstärkte - zuerst im Süden und Südosten, dann aber auch in den Nordprovinzen. Nach der schrittweisen Ausdehnung des ISAF-Mandats von der Hauptstadt Kabul auf das ganze Land beschloss die Bundesregierung, das deutsche ISAF-Kontingent solle im vergleichsweise ruhigen Norden stationiert werden. Jedoch erwies sich diese Lagebeurteilung als voreilig, denn auch im Norden nahmen Überfälle auf Bundeswehr-Soldaten, zivile Hilfsorganisationen und Regierungseinrichtungen ab 2006 stetig zu. Darauf war die Bundeswehr nicht oder ungenügend vorbereitet, weder von der Einstellung ihres Führungspersonals noch von der materiellen Ausstattung und Bewaffnung her gesehen.

Mit der wachsenden Zahl von verwundeten und getöteten deutschen Soldaten ${ }^{19}$ wurden die Fragen nach den Zielen, dem Sinn, der Organisation des deutschen ISAF-Einsatzes drängender. Die Berichterstattung in den Medien wurde aufmerksamer und kritischer. Erfahrungsberichte von Soldaten erschienen, in denen oft heftige Kritik an den Politikern in Berlin, an der Bundeswehr-Führung und an der öffentlichen Debatte oder eigentlich: Nicht-Debatte geübt wurde. ${ }^{20}$ Selbst die

18 Barfield, T.: Afghanistan. A Cultural and Political History, Princeton, N. J., 2010, 277.

1943 deutsche Soldaten und 3 deutsche Polizisten sind bisher (Stichtag: 15. 4. 2010) während der ISAFMission getötet worden, davon 29 unter gewaltsamen Umständen.

20 Vgl. etwa: Wohlgethan, A./Schulze, D.: Endstation Kabul. Als deutscher Soldat in Afghanistan - ein Insiderbericht, Berlin, 2008; Lindemann, M.: Unter Beschuss. Warum Deutschland in Afghanistan scheitert, Berlin, 2010. 
öffentliche Sprache für die Vorgänge in Afghanistan hinkte hinter der Wirklichkeit her. Erst 2008 sprach der damalige Verteidigungsminister Jung von den Getöteten als Gefallene ${ }^{21}$. Und bis ins Jahr 2010 blieb es umstritten, ob es sich bei den Kämpfen, in welche die Bundeswehr im Norden Afghanistan verwickelt wurde, um eine reine Stabilisierungsmission, um kriegsähnliche Zustände oder schlicht um Krieg handelt. Bis dahin hatte sich die Bundeswehr nur einmal an einem Krieg beteiligt, den Kosovo-Einsatz der NATO vom 24. März bis 10. Juni 1999. Aber dabei war es ausschließlich um Lufteinsätze gegangen. Jetzt hingegen gab es Selbstmordattentate, Hinterhalte und Überfälle ,in der Fläche“. Die Bundeswehr war darauf vorbereitet, zivile Aufbauprojekte zu schützen, zuweilen sich selbst daran zu beteiligen, afghanisches Personal auszubilden. Auf einen Krieg war sie nicht vorbereitet.

\section{Ein Scherbenhaufen}

Bundeswehrführung und deutsche Öffentlichkeit haben bis 2009 einige Anstrengungen darauf verwendet, die drohenden Anzeichen der Gewalteskalation zu verdrängen. In diesem Jahr kam es dann, teils geplant, teils überraschend, zu einer Zäsur - sowohl was den Afghanistan-Einsatz, als auch was die Bundeswehr-Reform angeht. Die Bundestagswahlen vom 27. September 2009 beendeten die Große Koalition aus CDU/CSU und SPD und brachten eine „schwarz-gelbe“ Regierungskoalition. Bei der Koalitionsverhandlungen über die Besetzung der Kabinettsposten ergab es sich, dass Karl-Theodor zu Guttenberg (CSU) anstelle von Franz Josef Jung (CDU) Verteidigungsminister wurde. Mit dieser Personalentscheidung kamen eine Reihe von Entwicklungen im Militärressort erst zur Sprache und dann ins Rollen, die bis zu diesem Zeitpunkt weder angesprochen noch bewegt werden sollten.

Allerdings gab es drei Wochen vor den Wahlen bereits einen dramatischen Vorfall in Afghanistan, der für die nächsten Wochen und Monate zu heftigen öffentlichen Kontroversen in Deutschland führen und der Bundeswehr-Reform einen kräftigen Anstoß geben sollte. Am 4. September 2009 wurden in der Nähe von Kunduz auf Befehl eines Bundeswehr-Offiziers zwei von den Taliban gekaperte Tanklaster bombardiert. Die Tanklaster hatten sich in einem Flussbett festgefahren, und es war unklar, ob die Menschen, die sich im Dunkeln um sie herum zu schaffen machten, Aufständische waren oder Zivilisten, die sich privat etwas 
Treibstoff abzapfen wollten. Als Folge der Bombardierung starben knapp 100 Afghanen, von denen Verteidigungsminister Jung zunächst behauptete, sie seien durchwegs Taliban-Kämpfer gewesen. Das stimmte aber nicht. Es folgte eine Reihe peinlicher Untersuchungen. In deren Verlauf ereigneten sich der Rücktritt von Minister Jung (zwischenzeitlich Minister für Arbeit und Soziales), die Entlassung von Generalinspekteur Schneiderhan und von Staatssekretär Wichert durch Minister $z u$ Guttenberg und die Einsetzung eines Parlamentarischen Untersuchungsausschusses. Der Ton der Kritik an der Bundeswehr-Führung, an der mangelhaften Ausrüstung der Bundeswehr in Afghanistan ${ }^{22}$ und am den von der Bundesregierung verkündeten Zielen des Einsatzes ${ }^{23}$ wurde rasch schärfer und bitterer. $^{24}$ Während aus der „Bevölkerungsumfrage 2008“ des Sozialwissenschaftlichen Instituts der Bundeswehr noch hervorgeht, dass drei von vier Befragten dem Afghanistan-Einsatz der Bundeswehr eher zustimmen ${ }^{25}$, drücken in einer Allensbach-Umfrage vom Mai 2010 zwei Drittel aller Befragten ihr Unbehagen an der deutschen Beteilung an ISAF aus ${ }^{26}$.

In einem Interview über die aktuellen Zustände in Afghanistan beantwortete Ahmed Rashid, einer der besten Kenner der Region die Frage „Wie beurteilen Sie die Rolle der Deutschen in Afghanistan?“ zunächst mit der Gegenfrage: „Wollen Sie die Antwort wirklich hören? Als die Interviewerin tapfer nickte, meinte er lapidar: „Deutschland ist nicht in der Lage, die Aufgaben zu erfüllen, die es übernommen hat...sehen Sie Kunduz an: Da wimmelt es heute von Taliban. Also was zum Teufel haben die Deutschen dort in den vergangenen fünf Jahren gemacht?

Bitterkeit ist auch ein Schlüsselwort für einen anderen Teil dieses zivilmilitärischen Scherbenhaufens. Die Einsätze auf dem Balkan, aber besonders der in Afghanistan waren bei immer noch relativ wenigen Soldaten die Ursache äußerer Verletzungen. Viel größer ist hingegen die Zahl der Soldaten mit psychischen Traumata. Die Medizin nennt solche seelischen Verletzungen Post-

22 Vgl. „Nicht immer sachgerecht und angemessen“, Frankfurter Allgemeine Zeitung, 17. April 2010.

23 Vgl. Frankfurter Allgemeine Sonntagszeitung, 31. Januar 2010.

24 Reichelt, J./Meyer, J.: Ruhet in Frieden, Soldaten! Wie Politik und Bundeswehr die Wahrheit über Afghanistan vertuschten, Köln 2010, besonders 121-152.

25 Frankfurter Allgemeine Zeitung, 13. November 2008.

26 Frankfurter Allgemeine Zeitung, 26. Mai 2010 - bei aller Vorsicht gegenüber solchen Zahlen kommt man nicht darum herum, einen deutlichen Meinungsumschwung in der Öffentlichkeit im Jahr $2009 \mathrm{zu}$ konstatieren.

27 Frankfurter Allgemeine Sonntagszeitung, 19. September 2010. 
traumatische Belastungsstörungen (PTBS). Die Bundeswehr ist diesem Leiden vieler ihrer Angehörigen allzu lange nur mit bürokratischer Gründlichkeit begegnet, mehr bürokratisch als gründlich allerdings und ohne die wünschenswerte Empathie für die Opfer. ${ }^{28}$

All dies zusammen und ein Einflussfaktor auf einer ganz anderen Ebene, nämlich die infolge der internationalen Finanzkrise prekäre Kassenlage des Staates ${ }^{29}$, haben bewirkt, dass der Reformprozess der Bundeswehr seit Ende 2009 nicht mehr im gemächlichen Tempo der vergangenen Jahre, sondern jetzt geradezu im Akkord vorangetrieben wird.

\section{Der erzwungene Reformsprung}

Allgemein kann man sagen, dass Zeiten mit finanziellen Engpässen sich gar nicht so schlecht dazu eignen, politische Reformen auf den Weg zu bringen. Denn sie zwingen die Verantwortlichen, sich über eine bessere Kosten-NutzenRelation Gedanken zu machen. Dieser Zwang muss nicht in jedem Fall, aber er kann gestalterische Kreativität befördern. Kommt noch wie in diesem Fall eine steigende Unzufriedenheit mit der Leistung einer teuren Organisation wie der Bundeswehr hinzu, liegt es auf der Hand, dass die üblichen und sonst meist präferierten Methoden wie das Durchwursteln (muddling through), das Einfach-soweitermachen (business as usual) und das Schönreden (public relations) nicht mehr ausreichen. Die deutsche Sicherheitspolitik, so Klaus Naumann ${ }^{30}$, sei ein Paradefall für Selbstsuggestion. „Die deutsche Politik lebt von der Illusion, mit den , bewährten' Institutionen und Prozeduren ließe sich auch unter völlig veränderten Bedingungen genauso gut fahren wie in den Jahren der guten alten Bonner Republik. “ ${ }^{31}$

Nicht zuletzt spielt bei dem neuen Tempo der Bundeswehr-Reform auch der Personal-Faktor eine Rolle. Franz Josef Jung machte oft den Eindruck, in seinem Amt überfordert zu sein. Sein Nachfolger Karl-Theodor zu Guttenberg hingegen

28 Vgl. Timmermann-Levanas, A./Richter, A.: Die reden Wir sterben. Wie unsere Soldaten zu Opfern der deutschen Politik werden, Frankfurt/M. 2010.

29 Vgl. Brune, S.-Ch./Dickow, M./Linnenkamp, H./Mölling, Ch.: Die Bundeswehr in Zeiten der Finanzkrise, Berlin 2010 (SWP-Aktuell 5/2010).

30 Namensgleich, jedoch weder identisch noch verwandt mit dem früheren Generalinspekteur der Bundeswehr.

31 Naumann, K.: Soldaten sollen denken, in: Die ZEIT, 4. Februar 2010. 
gilt als energisch und ehrgeizig und strebt deshalb auch auffällig danach, der schwerfälligen Militär-Organisation seine Prägung aufzudrücken. ${ }^{32}$

\section{Professionellere Streitkräfte}

Nachdem die deutsche Sicherheitspolitik in der zweiten Hälfte des Jahres 2009 und auch noch den ersten Monaten von danach vor allem im Schatten der ,Tanklaster-Affäre" mit anschließenden Schuldzuweisungen und personellen Konsequenzen gestanden hatten, kam im Frühjahr 2010 der neue Reform-Anlauf in die Spur. Der Minister setzte am 12. April eine „Strukturkommission“ ein, mit nur sechs Mitgliedern knapp und weniger diskurs- als ergebnisorientiert besetzt. Von dieser Kommission werden bis Ende 2010 weitgehende, aber zugleich auch handhabbare Vorschläge für eine Straffung der Führungs- und Verwaltungsstrukturen der Bundeswehr erwartet.

Am 26. Mai kündigte $z u$ Guttenberg in einer Rede an der Führungsakademie in Hamburg an, dass die Bundeswehr mit tiefen finanziellen Einschnitten fertig werden müsse und sich deshalb auf umfangreiche und schonungslose Veränderungen einzustellen habe. Am 7. Juni beschloss das Bundeskabinett einen „Prüfauftrag“ für das Verteidigungsministerium. Geprüft werden soll die Optimierung der Struktur der Bundeswehr in Hinsicht auf das von ihr erwartete EinsatzFähigkeits-Profil. Ende Juni wurden dann die „Leitlinien zur Ausplanung der neuen Bundeswehr" veröffentlicht, gewissermaßen die ausformulierten Rahmendaten für das bis spätestens Ende September fällige Ergebnis des Prüfauftrags. Anfang September lag der 62seitige „Bericht des Generalinspekteurs der Bundeswehr zum Prüfauftrag aus der Kabinettsklausur vom 7. Juni 2010“ vor und bildet nunmehr die Grundlage für die Überlegungen und Maßnahme im Ministerium und im Parlament zur Reform der Bundeswehr.

Diese Reform betrifft nicht nur die Bundeswehr, sondern auch die Sicherheitspolitik insgesamt, und es wird ausländische Beobachter mit einer hohen Meinung von der deutschen Neigung zu Gesamtkonzeptionen freuen, dass der Generalinspekteur Volker Wieker (im Amt seit dem 21. Januar 2010) den Reformprozess

32 Das ist allerdings ein ambitioniertes Ziel. In vergleichender organisations-soziologischer Betrachtung hat das Verteidigungsministerium den Ruf, besonders schwer regierbar zu sein. Konnten sich in der Frühzeit der Bundeswehr Franz Josef Strauß und später dann Helmut Schmidt (beide im übrigen auch militärstrategisch bewandert) im Umgang mit der Ministerialbürokratie und der Generalität hervorragend behaupten, lässt sich das von den meisten anderen Verteidigungsministern (Blank, von Hassel, Schröder, Leber, Apel, Wörner, Scholz, Stoltenberg, Rühe, Scharping, Struck, Jung) nur mit - unterschiedlich großen - Abstrichen feststellen. 
in einen „ganzheitlichen Ansatz“ einordnet (S. 7). Nach ein paar den Status quo resümierenden Einleitungen kommt der Bericht allerdings in deutlicher Sprache zur Sache. Die quasi-permanente Reform der Bundeswehr in den letzten zwei Jahrzehnten wird sprachlich zu einem „Anpassungsprozess“ (S. 12) herabgestuft. Jetzt bedürfe es weiterer substanzieller Schritte, um die Bundeswehr zukunftsfähig zu gestalten. Bei dieser Um- oder Neugestaltung ,ist eine Schwerpunktverlagerung zu professionelleren Streitkräften sicherheitspolitisch unabdingbar" (S. 12). Die Einsatzfähigkeit unter Bedingungen, wie sie etwa in Afghanistan bestehen, soll vergrößert werden. Als „wahrscheinliche Einsatzformen“, auf welche die Bundeswehr sich vorbereitet zeigen muss, werden aufgeführt: friedensstabilisierende Operationen in Anlehnung an laufende Einsätze; schnellere Reaktionen zur Unterstützung von Bündnispartnern; friedensschaffende Operationen; Verstärkung von Bündniskräften in Fällen einer länger dauernden, sich zuspitzenden Krise; Rettung und Evakuierung deutscher Staatsbürger aus Gefahrensituationen im Ausland; Maßnahmen zur Landesverteidigung für die Sicherheitsvorsorge in Deutschland; subsidiäre Hilfeleistungen in Deutschland (S. 22). Um dies gewährleisten zu können, müssen die Zielvorgaben für die Bundeswehr angehoben werden. Sie muss fähig sein zu Dauereinsätzen mit mindestens 10.000 (statt bislang nur rund 7.000) Soldatinnen und Soldaten in mehreren Einsatzgebieten, zur Vollunterstützung der Anteile Land- und Luftstreitkräfte in zwei Einsatzgebieten sowie zu entsprechender Unterstützung der Seestreitkräfte (S. 24).

\section{Alternativmodelle}

Gegenwärtig verfügt die Bundeswehr über 250.000 Soldatinnen und Soldaten. Davon sind 195.000 Berufssoldaten und länger dienende Soldaten auf Zeit (mit unterschiedlich langen Verpflichtungszeiten), 30.000 Grundwehrdienst Leisten$\mathrm{de}^{33}$ sowie 25.000 freiwillig zusätzlichen Wehrdienst Leistende ${ }^{34}$. Gegliedert sind sie in drei Kräftekategorien: Eingreifkräfte (35.000), Stabilisierungskräfte (70.000) und Unterstützungskräfte (145.000). Diese Struktur wird im Bericht des Generalinspekteurs „,ineffektiv“ genannt. Eine kleinere Bundeswehr, die aber

33 Die Dauer des Grundwehrdienstes schwankte in den vergangenen Jahrzehnten nicht unerheblich. Ab dem 1. Juli 2010 beträgt sie nur noch sechs Monate.

34 Als freiwillig zusätzlichen Wehrdienst Leistende (FWDL) werden Wehrpflichtige bezeichnet, die sich nach Ablauf ihrer Grundwehrdienstzeit noch weiter verpflichten, was bis zu insgesamt 23 Monate Wehrdienst möglich ist. Sie erhalten einen höheren Wehrsold und müssen sich auch verpflichten, unter Umständen an einem Auslandseinsatz der Bundeswehr teilzunehmen. Nimmt man es genau, handelt es sich bei ihnen nicht um wehrpflichtiggemäß, sondern um freiwillig dienende Soldaten. 
quantitativ und qualitativ einsatzfähiger ist, das ist die Reform-Aufgabe. In den Planungen des Ministeriums werden fünf Modelle aufgestellt und miteinander verglichen. Entscheidende planerische Vorgabe ist dabei, die Zahl der Berufssoldaten und Soldaten auf Zeit um 40.000 zu kürzen, zugleich aber die Einsatzfähigkeit der Bundeswehr zu erhöhen.

Das Modell 1 rechnet mit dieser Reduzierung in zwei Jahresschritten. Zusätzlich soll die Anzahl der Grundwehrdienstleistenden um 5.000 Soldaten gekürzt werden. Die Zahl von freiwillig zusätzlichen Wehrdienst Leistenden bleibt gleich.

Das Modell 2 rechnet mit dem Wegfall von Grundwehrdienst und freiwillig zusätzlichen Wehrdienst Leistenden und mit einer Reduzierung des Umfangs der dann also ausschließlich aus Berufssoldaten und Soldaten auf Zeit bestehenden Bundeswehr auf 150.000 Soldatinnen und Soldaten.

Das Modell 3 streckt den Abbau von Berufssoldaten und Soldaten auf Zeit über sechs Jahre und kürzt deren Zahl nur um 39.000. Grundwehrdienstleistende und freiwillig zusätzlichen Wehrdienst Leistende fallen in diesem Modell ebenfalls ganz weg, so dass der Streitkräfteumfang im Jahr 2016 insgesamt 156.000 Soldatinnen und Soldaten beträgt.

Das Modell 4 nimmt dieselben Kürzungen wie das Modell 3 vor. Es führt allerdings eine neue Kategorie von Soldaten ein: solche die einen Freiwilligen Wehrdienst leisten. Der Gedanke dahinter ist der: Wenn die Wehrpflicht entfällt, könnten dennoch eine Reihe junger Männer und gegebenenfalls neu hinzukommend auch jüngere Frauen Interesse am Soldatenberuf bekunden, ohne sich sogleich auf mehrere Jahre verpflichten zu wollen. Für diese Kategorie von Dienstleistenden sind ab 2013 insgesamt 7.500 Stellen vorgesehen. Der Streitkräfteumfang im Jahr 2016 beträgt in diesem Modell 163.500 Soldaten und Soldatinnen.

Das Modell 5 schließlich senkt die Zahl der Berufssoldaten und Soldaten auf Zeit nur um 15.000, lässt dafür aber die Kategorie der freiwillig zusätzlichen Wehrdienst Leistenden gänzlich fallen. Die Zahl der Grundwehrdienst Leistenden bleibt mit 30.000 konstant, so dass sich insgesamt ein Streitkräfteumfang von 210.000 Soldatinnen und Soldaten ergibt.

\section{Implikationen}

In der berichts-internen Abwägung der Vor- und Nachteile, die mit den fünf Modellen verbunden sind, kommt der Generalinspekteur zu folgendem Ergebnis: 
Das Modell 1 benötigt eine beachtlich große Zahl von Soldaten für die Ausbildung der Wehrpflichtigen, so dass hier bis zu 125.000 Soldatinnen und Soldaten für Auslandseinsätze einplanbar sind. Das Potential der Grundwehrdienst und freiwillig zusätzlichen Wehrdienst Leistenden macht die Nachwuchswerbung vergleichsweise einfach.

Das Modell 2 impliziert den Fortfall einer Reihe wichtiger militärischer Fähigkeiten der Streitkräfte und könnte dadurch nicht zuletzt auch die multilaterale Kooperation mit verbündeten Streitkräften gefährden. Bis zu 120.000 Soldatinnen und Soldaten wären für Auslandseinsätze einplanbar. Aber in mittlerer und längerer Perspektive würde der Fortfall der Wehrdienst Leistenden die Fähigkeit zur Rekonstitution $^{35}$ arg einschränken. Die Einsparungsvorgaben des Kabinetts würden mit diesem Modell am leichtesten erreichbar sein.

Das Modell 3 ist eine Art sanftere Version des Modells 2, weil der Abbau der Zahl der Berufssoldaten und Soldaten auf Zeit über längere Zeit gestreckt und nicht 45.000, sondern nur 39.000 Stellen umfasst. Damit wären bis zu 126.000 Soldatinnen und Soldaten für Auslandseinsätze einplanbar.

Das Modell 4 ist der Favorit des Generalinspekteurs. Es kommt auf eine Zahl von 133.500 für Auslandseinsätze einplanbare Soldatinnen und Soldaten, weil es einen „Freiwilligen Wehrdienst“ einführt, ein Instrument zur Erleichterung der Nachwuchswerbung, von dem allerdings noch niemand so recht sagen kann, ob es auch wirklich attraktiv genug ist. Der Gesamtumfang der Bundeswehr beträgt in diesem Modell 163.500 Soldaten, wobei allerdings „gesamtstaatliche Verpflichtungen wie Parlamentarischer Flugbetrieb und Sportförderung sowie ministerielle Aufgaben“ (S. 40) in diesem Umfang nicht mit abgedeckt sind.

Das Modell 5 würde ähnliche Auswirkungen haben wie das Modell 4; aber es würde mehr Kosten verursachen.

\section{Sachzwang und Lagebeurteilung}

Modelle sind ein Planungsinstrument, weil damit Erkenntnisse arrangiert und in ein übersichtliches Design gebracht werden können. Nützlich ist das, aber auch ein bisschen problematisch, weil das Denken in Modellen Übersichtlichkeit

35 Mit diesem Fachbegriff wird die Wiederherstellung früher vorhandener, jedoch zwischenzeitlich aufgegebener militärischer Potentiale bezeichnet. Die Forderung nach Aufrechterhaltung der Fähigkeit zu (möglichst rascher) Rekonstitution wird insbesondere von denjenigen betont, die eine direkte territoriale Bedrohung Deutschlands in der Zukunft als ein vielleicht nicht besonders vordringliches, aber durchaus realistisches Szenario ansehen. 
verspricht, die sich aber weitgehend verflüchtigt, je näher man hinsieht. Die Reformvorschläge Guttenbergs und seines Generalinspekteurs gründen sich auf ein Fundament aus Sachzwängen und Lagebeurteilungen, wobei der zwingende Charakter ersterer auch als Mittel der politischen Durchsetzung benutzt und ansonsten teilweise überspielt werden kann und die Schlüssigkeit letzterer sicherheitspolitisch nicht unstreitig ist.

Als Sachzwang stellt sich die finanzielle Situation des Bundes mit den enormen Belastungen des Haushalts aus der Finanzkrise 2008/2009 und wegen der 2009 ins Grundgesetz eingebauten „Schuldenbremse“ ${ }^{๔ 6}$ dar. Gewiss wirkt sich dieser Sachzwang als Zwang zum Sparen aus. Aber bei welchen Ausgaben wie drastisch gespart wird, bleibt zunächst einmal offen. Außerdem sind alle Prognosen über die Einnahmen der öffentlichen Hand in den nächsten Jahren von Unsicherheit überschattet - sie könnten günstiger oder ungünstiger ausfallen als erwartet. Dennoch lässt sich gut nachvollziehen, dass der Minister den Spardruck als reform-antreibendes Instrument benutzt. Das gehört im Übrigen genauso zu den parlamentarischen Spielregeln wie das Verhalten der Opposition, die in ihren Beiträgen zur Bundeswehr-Reform solchen Spardruck dezidiert ignoriert. Das auch bei Abgeordneten aus der Regierungskoalition vernehmbare Argument, es dürfe aber keine „Bundeswehr nach Kassenlage“ geben, ${ }^{37}$ wurde auch vom Minister selbst aufgegriffen.

Dieses Argument lässt sich im Streit innerhalb der Regierung um die Größe und Verteilung der Sparauflagen wegen seiner Popularität in der Öffentlichkeit gut einsetzen. Es stimmt auch, wenn man diese Ebene der konkreten Entscheidungsfindung über Haushaltszahlen, mittelfristige Finanzplanung und Sparziele betrachtet. Grundsätzlich aber stimmt es freilich nicht - die Höhe sämtlicher Ausgaben privater und öffentlicher Haushalte hängt selbstverständlich von der Kassenlage an, und es wäre nur der Ausdruck einer abenteuerlichen Politik, wenn es zu Ausgabenbeschlüssen käme, ohne dass die Kassenlage berücksichtigt wird. Diese Aussage gilt für alle Ressorts der Bundesregierung und nicht allein für die Sicherheitspolitik. Die ist aber, in eigentümlicher Parallelität zur Kulturpolitik, vergleichsweise mehr als andere Politikfelder dem Sachzwang der Kas-

36 Siehe Art. 109 (3) und Art. 115 Grundgesetz - die „Schuldenbremse“ bedeutet, dass die Haushalte des Bundes und der Länder ab 2016 ohne Kredite auszugleichen sind und die strukturelle Nettokreditaufnahme auf höchstens $0,35 \%$ des Bruttoinlandprodukts beschränkt bleiben muss. Vermutlich war diese Grundgesetzänderung, um die Worte Bundeskanzlerin (aus einem anderen Zusammenhang) zu zitieren, „nicht sehr hilfreich“.

37 So etwa Jörg van Essen (FDP) in einem Interview am 24. August 2010 im Deutschlandradio Kultur. 
senlage ausgesetzt, weil die Festlegung dessen, was optimal oder minimal für „unsere Sicherheit“ aufgebracht werden soll, von Bedrohungswahrnehmungen abhängt, die sich nicht eindeutig in quantitative Größen übersetzen lassen. Viele Beobachter der Sicherheitspolitik Deutschlands, die man nicht alle als Lobbyisten der Bundeswehr oder der Rüstungsindustrie kennzeichnen kann, halten etwa die Bundeswehr seit Jahren für unterfinanziert. Das heißt nichts anderes, als dass zwischen dem Auftrag der Bundeswehr und den ihr zur Verfügung gestellten Mitteln zur bestmöglichen Auftragserfüllung eine Lücke klafft. Diese Lücke kann man schließen, indem man entweder den Auftrag reduziert oder die Mittel erhöht.

Die Lagebeurteilung und die Einschätzung der Sicherheits-Landschaft mit ihren künftigen Veränderungen sind erheblich volatiler als die finanziellen Sparzwänge. Der Afghanistan-Schock hat jedenfalls bewirkt, dass sich das Anforderungsprofil für die Bundeswehr-Soldaten verschoben hat, und zwar in Richtung auf den „Kämpfer“-Typ ${ }^{38}$ in Situationen asymmetrischer Kriegsführung. Das ist unstrittig in und zwischen den Parteien im Bundestag und in der informierten Öffentlichkeit.

Durchaus umstritten ist demgegenüber die Frage nach dem Gewicht herkömmlicher Bedrohungen, also solcher, die einen direkten Angriff auf das eigene oder das Territorium der Verbündeten implizieren. Die in dem Übersichtskapitel über die Bundeswehr-Reformen der letzten beiden Jahrzehnte konstatierte Sowohl-als auch-Politik der verschiedenen Bundesregierungen lässt sich heute aber kaum noch fortführen. Anders gesagt: die Bundesregierung muss entscheiden, ob es den deutschen Interessen auch längerfristig eher entspricht, wenn die Bundeswehr weiterhin als Instrument deutscher Beteiligung an Weltordnungspolitik und Krisenstabilisierungen im Kontext von Vereinten Nationen, NATO, OSZE und Europäischer Union eingesetzt wird oder ob sie sich vor allem auf einen Verteidigungsauftrag in Mitteleuropa vorbereiten soll. Je nachdem braucht es unterschiedliche militärische Strukturen und Ausbildungsgrundsätze sowie andere Schwerpunkte bei der Bewaffnung und Ausrüstung. Beides nebeneinander geht aber immer schlechter.

38 Vgl. von Bredow, W.: Kämpfer und Sozialarbeiter. Soldatische Selbstbilder im Spannungsfeld herkömmlicher und neuer Einsatzmissionen, in: Gareis, S. B./Klein, P.: Handbuch Militär und Sozialwissenschaft, Wiesbaden 2006², 314-321; Herberg-Rothe, A.: Demokratische Krieger, in: Frankfurter Rundschau, 23. 7. 2009. 


\section{Dreh- und Angelpunkt Wehrpflicht}

Dreh- und Angelpunkt des gegenwärtigen Reformschubs bei der Bundeswehr ist die Frage nach der Beibehaltung oder Abschaffung/Aussetzung der Wehrpflicht. Hier überschneiden sich finanzielle, sicherheitspolitische und gesellschaftspolitische Aspekte. Hier treffen auch Pro- und Contra-Argumentationslinien aufeinander, die in den letzten Jahren immer erneut gegeneinander ins Feld geführt wurden. Dabei behielten die Befürworter der Wehrpflicht bislang immer die Oberhand, was aber weniger an der Durchschlagskraft ihrer Argumente, vielmehr an einem ausgeprägten institutionellen und organisatorischen Beharrungsvermögen lag. Die beiden großen Parteien im Bundestag CDU/CSU und SPD, die Bundeswehr-Führung, der Bundeswehrverband, die meisten Sozialverbän$\mathrm{de}^{39}$, indirekt auch das Bundesverfassungsgericht (erstaunlicherweise!) haben mit unterschiedlichen Akzentuierungen die Beibehaltung der Wehrpflicht für finanziell günstig, sicherheitspolitisch notwendig, staatspolitisch wertvoll, verfassungsrechtlich unbedenklich sowie gesellschaftspolitisch fair beurteilt und die Abschaffung/Aussetzung der Wehrpflicht für demokratie-gefährdend, zu teuer sowie rekrutierungs-feindlich in Bezug auf die personelle Entwicklung der Bundeswehr. Ja, es ist sogar argumentiert worden, Berufsarmeen ließen sich leichter in den Krieg schicken als Wehrpflichtarmeen, wofür es in der deutschen Geschichte des 20. Jahrhunderts nun wirklich keinerlei Beleg gibt, weder 1914 noch 1939. Außerdem drückt sich in diesem Argument ein unausgesprochenes Misstrauen gegen die demokratischen Sicherungen aus, die nach dem Grundgesetz und weiteren gesetzlichen Bestimmungen einen leichtfertigen Einsatz der Bundeswehr verhindern.

Noch bis Anfang 2010 hat es eine große Zahl publizistischer Versuche gegeben, die Wehrpflicht zu „retten“. ${ }^{40}$ Ein grundlegendes empirisches Problem dabei drängt sich seit vielen Jahren ins Auge, nämlich dass die Bundeswehr schon lange alles andere als eine „Wehrpflichtarmee“ ist, denn Wehrpflichtige machen nur einen geringen Prozentsatz der Soldatinnen und Soldaten aus, ein Fünftel ungefähr. Außerdem macht der Anteil der einberufenen Wehrpflichtigen nur einen kleinen Teil der Jahrgangsstärke junger Männer aus - seit 2007 weniger als

39 Diese sind nicht an der Wehrpflicht, aber am Zivildienst interessiert. Der muss bei einer Aussetzung der Wehrpflicht automatisch entfallen.

40 Vgl. etwa: Kirsch, U. (Hg.): Darum Wehrpflicht! Zur aktuellen Debatte um die Zukunft der deutschen Wehrpflicht, Baden-Baden 2010; Ahammer, A./Nachtigall, S. (Hg.): Wehrpflicht - Legitimes Kind der Demokratie, Berlin 2010. 
ein Fünftel. ${ }^{41}$ Gesundheitliche Gründe bei der Musterung, Inanspruchnahme des Rechts auf Kriegsdienstverweigerung und Ausnahmeregelungen spielen dabei eine Rolle. Selbstverständlich könnten durch Änderungen bei den MusterungsKriterien mehr wehrpflichtige junge Männer auch eingezogen werden. Aber erstens ist fraglich, ob die Bundeswehr einen entsprechenden Bedarf hat, und zweitens würde das politisch-rechtlich-ideologische Problem der mangelnden Wehrgerechtigkeit dadurch nicht wesentlich gemildert. Alle sozio-politischen Gründe für die Beibehaltung der Wehrpflicht halten einer Prüfung nicht stand. Die Integration der Bundeswehr in die Gesellschaft kann mittels 70.000 oder 80.000 Wehrpflichtige pro Jahr, die bis vor kurzem neun, jetzt nur noch sechs Monate „beim Bund“ ihren Dienst leisten, nicht besser garantiert werden als durch eine Freiwilligen-Streitkraft, bei deren Ausbildung darauf geachtet wird, dass die Innere Führung und das Leitbild des Staatsbürgers in Uniform im individuellen und kollektiven Selbstverständnis der Soldaten einen zentralen Platz einnehmen. Ein Satz wie der folgende ist heute unverständlich: „Mit jedem Wehrpflichtigen übt die Bevölkerung Kontrolle darüber aus, ob den Grundsätzen unserer Verfassung entsprochen wird. “42 Für die Anfangsjahre der Bundeswehr und unter den politischen Auspizien des Ost-West-Konflikts hatte er seine Berechtigung. Insofern hat sich die Wehrpflicht als Wehrform unter anderen Verhältnissen sehr wohl bewährt. Dies festzustellen, ist kein Widerspruch zu der These, dass sie nach dem „Übergang von der Landesverteidigung zur Sicherheitsvorsorge “433 funktional überholt ist.

Hier nun hat Minister zu Guttenberg in klarer Abgrenzung zu seinen Amtsvorgängern einen anderen Weg eingeschlagen. „Der Mehrwert der allgemeinen Wehrpflicht besteht heute vorrangig in ihrem Beitrag zur Nachwuchsgewinnung", heißt es lakonisch im Bericht des Generalinspekteurs, General Wieker, vom September 2010 (S. 18). Spätestens die gerade erst beschlossene Verkürzung des Grundwehrdienstes auf sechs Monate hat diesen sowieso zu einer Art von verlängertem Praktikum herabgestuft. Dass er als solches für die Nachwuchsgewinnung der Bundeswehr nützlich ist, braucht nicht in Frage gestellt zu werden. Jedoch weisen weder die sicherheitspolitischen noch die gesellschaftspolitischen Argumente genügend Überzeugungskraft auf, um eine inzwischen

41 Frankfurter Allgemeine Zeitung, 15. September 2010.

4250 Jahre Wehrpflicht in der Bundeswehr. Namensartikel des Generalinspekteurs der Bundeswehr, General Wolfgang Schneiderhan, in: Bundeswehr aktuell, 30. März 2007.

43 Naumann, K.: Einsatz ohne Ziel? Die Politikbedürftigkeit des Militärischen, Hamburg, 2008, 21. 
auf geradezu kapriziöse Weise selektiv gewordene Institution wie die allgemeine Wehrpflicht für Männer zu rechtfertigen. Zumal mit einem kreativen Rekrutierungs-Management und neuartigen Institutionen wie dem Freiwilligen Wehrdienst ein funktionales Äquivalent für diesen Mehrwert der Wehrpflicht bereitgestellt werden kann. ${ }^{44}$

Ein nun allerdings auch schwer ins Gewicht fallendes Argument, das sich heute für die Beibehaltung der Wehrpflicht vorbringen lässt, lautet: Nur so lassen sich genügend Reservisten produzieren, auf die im Fall der Notwendigkeit zur Rekonstitution zurückgegriffen werden kann. Das Modell 4 des Ministers, für das er seit Anfang September 2010 in CDU und CSU erfolgreich geworben hat, ${ }^{45}$ wird, falls umgesetzt, die Fähigkeit zur Rekonstitution erheblich einschränken. Im Grunde lautet also die grundsätzliche sicherheitspolitische Frage, deren Beantwortung über Beibehaltung oder Abschaffen/Aussetzen ${ }^{46}$ der Wehrpflicht entscheiden sollte: Wie sieht die Priorität bei den militärischen Aufgaben der Bundeswehr aus und wie hoch ist die Wahrscheinlichkeit, dass sich Deutschland in den nächsten beiden Jahrzehnten gegen einen massiven militärischen Angriff auf sein Territorium verteidigen muss?

Nur wenn man, wie offenbar Minister zu Guttenberg und seine obersten militärischen Berater im Einvernehmen mit den entsprechenden Lagebeurteilungen der NATO diese Wahrscheinlichkeit als sehr gering ansieht und das auch überzeugend $\mathrm{zu}$ begründen versteht, ist die Abschaffung/Aussetzung der Wehrpflicht eine angemessene Reform-Maßnahme. Für Lothar Rühl, einen der ausgewiesensten Kenner der deutschen Militär- und Sicherheitspolitik, ist genau das aber realistisch: „Es kann keinen Rückzug auf die territoriale Landes- und Bündnisverteidigung in Europa geben. “47

44 Sehr ausgewogen und mit konstruktiven Vorschlägen zur ,Trias von Freiwilligenarmee, Bürgerreserve und freiwilligem Gemeinschaftsdienst“: Werkner, I.-J.: Noch die Schule der Nation?, in: Frankfurter Allgemeine Zeitung, 23. Juli 2010.

45 Vgl. u. a.: Süddeutsche Zeitung, 11. September 2010: „Seehofer will Wehrpflicht abschaffen“; ZEITOnline, 11. September 2010: „Auch die Hessen-CDU will ihren Widerstand aufgeben“.

46 Der Unterschied zwischen diesen beiden Maßnahmen ist graduell. Das Aussetzen der Wehrpflicht bedeutet, dass alle gesetzlichen Regelungen bestehen bleiben, welche es ermöglichen, Wehrpflichtige einzuberufen. Nur in der Praxis wird davon kein Gebrauch gemacht. Auch einen Teil der organisatorischen Instrumente zur Einberufung könnte man aufrechterhalten, wenn auch in reduzierter Form (z. B. Kreiswehrersatzämter). Das ist auch eine Kostenfrage. Auf jeden Fall ist es politisch klüger, die Wehrpflicht erst einmal auszusetzen und nicht gleich radikal abzuschaffen. Beispiele dafür gibt es im Übrigen auch bei anderen NATO-Mitgliedsstaaten.

47 Rühl, L.: Was die Bundeswehr braucht. Anmerkungen zum Bericht des Generalinspekteurs, in: Frankfurter Allgemeine Zeitung, 24. September 2010. 


\section{Fazit}

Anders als in der Außenpolitik sind den Bundesregierungen und der Öffentlichkeit in Deutschland die neuen Herausforderungen in der Sicherheitspolitik über einen längeren Zeitraum hinweg fremd und gewissermaßen unheimlich geblieben. Die Auslandseinsätze der Bundeswehr, in keinem Fall Ausdruck von irgendwelchen abenteuerlichen Motiven, wurden mit Vorliebe als kampflose Friedens- und Stabilisierungsmissionen wahrgenommen. Das war immer schon eine unangemessene Wahrnehmung, zugleich eine Illustration der These von der Persistenz lange und gerne gehegter Perzeptionen. Die Reform der Bundeswehr wurde versuchsweise als eine Art Marathon der kleinen Schritte in Angriff genommen - mit mageren Ergebnissen. Das führte nicht zuletzt auch zu Irritationen bei den Verbündeten. Erst mit dem ISAF-Einsatz im Norden Afghanistans und den sich seit 2005 mehrenden und verschärfenden Kampfsituationen begann eine öffentliche Debatte über Aufgaben und Profil der Bundeswehr, die allerdings immer noch um die Härten und die Gefährlichkeit der neuen Stabilisierungs- und Krisenreaktions-Missionen rhetorische Bögen machte. Der Schock, den im September 2009 die von einem Offizier der Bundeswehr befohlene TanklasterBombardierung in der Nähe von Kundus in der deutschen Öffentlichkeit auslöste, die steigende Zahl getöteter und physisch und psychisch verletzter Bundeswehr-Soldaten in Afghanistan und die von der Bundesregierung gezogenen Folgerungen aus der Finanzkrise 2008/2009 für ihre Haushaltspolitik der kommenden Jahre wirkten zusammen, um den seit Ende Oktober 2009 amtierenden Verteidigungsminister zu Guttenberg von der Methode der kleinen Reformschritte abgehen zu lassen und einen kräftigen Reformsprung ins Auge zu fassen.

Zum Teil gegen anfängliche Widerstände in der CDU/CSU begonnen und derzeit noch nicht abgeschlossen, wird dieser Reformsprung eine Verkleinerung des Umfangs der Bundeswehr bewirken, eine Erhöhung der Zahl der für Auslandseinsätze geeigneten Soldatinnen und Soldaten, eine Professionalisierung des Berufsbildes der Soldaten, eine einschneidende Reform der zivilen Teile der Bundeswehr (Bundeswehrverwaltung), eine Verbesserung der innerministeriellen Kommunikations- und Befehlswege, vielleicht auch eine gründliche Organisationsreform der Teilstreitkräfte und ihrer wechselseitigen Bindungen. Am Ende dieser Reformsequenz wird es keine Wehrpflicht mehr geben, wenn auch die rechtlichen Grundlagen zu ihrer Wiedereinführung bestehen bleiben werden.

Reformen wecken Erwartungen. Nach ihrer Umsetzung soll alles besser werden. Häufig sind solche Erwartungen zu hoch, weil sie, um sie besser in der Öffent- 
lichkeit „verkaufen“ zu können, von ihren Protagonisten gerne zu einseitig dargestellt werden. Außerdem schaffen Reformen, indem sie alte Probleme überwinden, zuweilen neue Probleme. Das wird auch mit den jetzt in Angriff genommenen Bundeswehr-Reformen nicht anders sein. Zwei skeptische Ausblicke sollen deshalb diese insgesamt zu einem positiven Fazit gelangende Analyse beschließen. Erstens wird es nicht wirklich gelingen, mit den vorgeschlagenen Veränderungen der Bundeswehr deutlich zu Buche schlagende Einspar-Effekte zu erreichen. Die Bundeswehr wird zwar durchaus effektiver, billiger aber wird sie nicht. Zweitens wird es noch erheblicher Anstrengungen bedürfen, um nach dem faktischen Ende der Wehrpflicht den Nachwuchs für die Bundeswehr rekrutieren zu können, auf den die Streitkräfte angewiesen sind. Das ist auch, aber nicht in erster Linie eine Frage des Geldes. Ob es gelingt, hängt hauptsächlich vom Wirkungsgrad der Integration von Bundeswehr und Gesellschaft ab, von der Außen- und Sicherheitspolitik, vom politischen Diskurs an der Spitze des Gemeinwesens, in den Medien und in der demokratischen Öffentlichkeit. 